\title{
Percepções da dor: diagnóstico de enfermagem em pacientes infartados
}

\author{
Perceptions of pain: diagnosis of nursing in infarcted patients
}

Percepciones del dolor: diagnóstico de enfermería en pacientes infartados

\begin{abstract}
Adilson Mendes de Figueiredo Júnior ${ }^{1 *}$, Mayara Melo Galvão², Jhonatan Pereira Souza³.
\end{abstract}
\section{RESUMO}

Objetivo: Conhecer as percepções do sintoma da dor para os pacientes diagnosticados com Infarto Agudo do Miocárdio (IAM). Métodos: Estudo descritivo e exploratório com abordagem qualitativa, e consistiu de pesquisa bibliográfica e entrevista dirigida e semiestruturada. Resultados: $62,5 \%$ (5) pacientes são do sexo feminino. $75 \%$ (6) pacientes se encontram na faixa etária acima de 60 anos. 3 pacientes relataram que a dor iniciou após realizarem atividades diárias e 3 a descreveram como se fosse algo que não tem explicação. Conclusão: A definição e o enfrentamento da dor é algo pessoal e exclusivo de cada indivíduo, mesmo que ambos passem por situações similares, cada um terá seu modo de expressar e descrever o significado da dor e o que ela representou no determinado momento que ocorreu.

Palavras-Chave: Enfermagem, Infarto, Diagnóstico.

\section{ABSTRACT}

Objective: To know the perceptions of the pain symptom for patients diagnosed with acute myocardial infarction (AMI). Methods: Descriptive and exploratory study with a qualitative approach, and consisted of bibliographical research and directed and semi - structured interviews. Results: $62.5 \%(5)$ patients are female. $75 \%$ (6) patients are in the age group above 60 years. 3 patients reported that the pain started after performing daily activities and 3 described it as something unexplained. Conclusion: The definition and coping of pain is something personal and exclusive to each individual, even though they both go through similar situations, each one will have his way of expressing and describing the meaning of pain and what it represented in the given moment that occurred.

Keywords: Nursing, Infarction, Diagnose.

\section{RESUMEN}

Objetivo: Conocer las percepciones del síntoma del dolor para los pacientes diagnosticados con Infarto Agudo del Miocardio (IAM). Métodos: Estudio descriptivo y exploratorio con abordaje cualitativo, y consistió de investigación bibliográfica y entrevista dirigida y semiestructurada. Resultados: $62,5 \%$ (5) pacientes son del sexo femenino. $75 \%$ (6) pacientes se encuentran en el grupo de edad superior a 60 años. 3 pacientes relataron que el dolor empezó después de realizar actividades diarias y 3 a describieron como si fuera algo que no tiene explicación. Conclusión: La definición y el enfrentamiento del dolor es algo personal y exclusivo de cada individuo, aunque ambos pasen por situaciones similares, cada uno tendrá su modo de expresar y describir el significado del dolor y lo que ella representó en el determinado momento que ocurrió.

Palabras-clave: Enfermería, Infarto, Diagnóstico.

\footnotetext{
1 Escola Superior da Amazônia (ESAMAZ), Belém-PA. * E-mail: adilsonmdfj@hotmail.com

2 Universidade do Estado do Pará (UEPA), Belém-PA.

3 Universidade da Amazônia (UNAMA), Belém-PA.
} 


\section{INTRODUÇÃO}

As doenças isquêmicas do coração são motivos frequentes de atendimento em unidades de emergência, sendo as síndromes coronarianas agudas (SCA) incluídas nesse grupo de doenças, como causas prevalentes de morbidade e mortalidade (FALCÃO, COSTA e AMARAL, 2010). Os principais fatores de risco para as SCA incluem: álcool, tabagismo, história pregressa de problema cardíaco, hipertensão arterial sistêmica, diabetes mellitus e uso de drogas ilícitas. Segundo a autora Francelino et al (2016),

O Infarto Agudo do Miocárdio (IAM) é uma das principais doenças relacionadas a isquemia do coração, e refere-se ao processo pelo qual áreas do tecido miocárdico são destruídas de maneira permanente por conta de uma diminuição do fluxo sanguíneo coronariano, por estreitamento ou obstrução de uma artéria do coração por acúmulo de placas de ateromas, o qual produz uma relação desproporcional entre a oferta de suprimentos, notadamente o oxigênio, e seu consumo pelo miocárdio, de modo a se configurar uma lesão irreversível, necrosante (OLIVEIRA e SILVA, 2010).

IAM consiste em uma patologia incapacitante e limitadora, uma vez que repercute de forma negativa no padrão de vida do ser humano vida. É a primeira causa de mortes no Brasil, de acordo com a base de dados do DATASUS, de Departamento de Informática do SUS, que registra cerca de 100 mil óbitos anuais devido à doença. A dor torácica é o principal sintoma associado ao IAM, que é descrito como uma dor súbita, sobre o esterno, constante e constritiva, que pode ou não se irradiar para várias partes do corpo, como a mandíbula, costas, pescoço e membros superiores, especialmente a face interna do membro superior esquerdo, e dispnéia (BRASIL, 2015).

Embora, a dor torácica que acomete pacientes com IAM seja considerada característica, a avaliação da dor segue como um desafio aos profissionais de saúde nos serviços de emergência hospitalar devido à subjetividade e dificuldades em sua mensuração. Apesar de sua subjetividade, avaliar a dor configura-se em uma tarefa essencial ao trabalho da Enfermagem à medida que é um sintoma importante para a investigação do quadro clínico do paciente, sendo necessário ao enfermeiro dispensar especial atenção uma vez que o ato de medir o fenômeno álgico requer observação, escuta atenta e, sobretudo, acreditar na queixa dolorosa que o paciente refere (VIEIRA, 2014).

Reafirmando a necessidade de considerar a dor como prioridade no tratamento, estudos revelam que a maioria dos pacientes que procuram os serviços de emergência refere algum tipo de dor. Neste sentido a sua avaliação correta durante o acolhimento é essencial para uma classificação na prioridade adequada. Referente à subjetividade da dor, os aspectos culturais, de expressão verbal, expressões corporais e alterações de comportamento devem ser considerados (OLIVEIRA e SILVA, 2010). O presente estudo teve como objetivo principal conhecer as percepções do sintoma da dor para os pacientes diagnosticados com Infarto Agudo do Miocárdio (IAM).

\section{MÉTODOS}

Para situar o tema no contexto atual de pesquisas realizou-se uma Revisão Integrativa sobre o Tema RIL. O estudo é do tipo descritivo e exploratório com abordagem qualitativa, envolve o uso de técnicas padronizadas de coleta de dados como questionário e observação sistemática. Por ser de natureza exploratória, envolve levantamento bibliográfico, entrevistas com pessoas e análise de exemplos que estimulem a compreensão. Assim como, a abordagem qualitativa considera a relação dinâmica entre o mundo real e o sujeito.

O local de realização da pesquisa foi na Fundação Pública Estadual Hospital de Clínicas Gaspar Viana FPEHCGV, localizado no município de Belém do Pará. O Hospital é referência em Psiquiatria, Cardiologia e Nefrologia, com missão de garantir atendimento ambulatorial e hospitalar de média e alta complexidade, sendo campo de prática do Curso de Residência Multiprofissional em Atenção a Saúde Cardiovascular.

Para desenvolver a pesquisa, foi selecionada uma clínica do referido hospital, sendo ela: Clínica Cardiológica, pelo fato dos pacientes internados, em sua maioria, possuírem diagnóstico médico de Infarto

REAS/EJCH | Vol.Sup.21 | e547 | DOI: https://doi.org/10.25248/reas.e547.2019 Página 2 de 9 
Agudo do Miocárdio, que tem como um de seus sinais e sintomas principais a dor torácica aguda e repentina, além de serem pacientes considerados clinicamente estáveis, estando então hábeis para responder as perguntas da pesquisa sem que haja interferência no quadro clínico dos mesmos.

Os sujeitos da pesquisa foram 8 (oito) pacientes infartados com amostra de conveniência, internados na Clínica Cardiológica. Foram incluídos na pesquisa os pacientes de ambos os sexos, maiores de 18 anos, que possuíam diagnóstico de Infarto Agudo do Miocárdio, clinicamente estáveis, conscientes e orientados e internados na Clínica Cardiológica. Foram excluídos da pesquisa os pacientes com co-morbidades e que possuíam dor torácica, mas não eram diagnósticados com Infarto Agudo do Miocárdio (IAM).

A coleta de dados foi realizada no período de novembro e dezembro de 2016, através de entrevista dirigida (apresenta um roteiro previamente estabelecido) e semiestruturada, que são feitas oralmente em ordem prévia, mas na qual o entrevistador pode acrescentar questões de esclarecimento ou instigar as respostas do entrevistado. A entrevista foi gravada com a permissão dos entrevistados (SILVA e SILVEIRA, 2008).

Segundo Bardin, a análise de conteúdo caracteriza-se por um conjunto de técnicas de análise, que permitem de forma prática e objetiva produzir inferências do conteúdo da comunicação, visando obter por procedimentos sistemáticos e objetivos a descrição do conteúdo das mensagens. Enquanto que, a análise temática para o autor, consiste em descobrir os núcleos do sentido que compõe uma comunicação cuja presença ou frequência signifiquem alguma coisa para o objetivo analítico visado.

O material produzido nas entrevistas foi transcrito na íntegra e organizado, segundo questões e respostas, para visualizar o todo do material. A partir daí o material foi exaustivamente lido, codificado, representado em quadros para identificar os núcleos de sentido, considerando a presença e frequência dos aspectos identificados no material como relevante para responder os objetivos do estudo. Daí emergiu categorias, que orientaram a interpretação e a discussão dos resultados que responderam a problemática da pesquisa.

O estudo desta pesquisa foi pautado segundo a Resolução №466/2012. A referida resolução incorpora, sob a ótica do indivíduo e das coletividades, os referenciais da bioética, autonomia, não maleficência, beneficência, justiça e equidade, dentre outros e visa a assegurar os direitos e deveres que dizem respeito aos participantes da pesquisa, à comunidade científica e ao Estado. Este projeto foi cadastrado na Plataforma Brasil e aprovado pelo Comitê de Ética em Pesquisa da Fundação Pública Estadual Hospital de Clínicas Gaspar Viana, sob o protocolo de número 1.739.315.

\section{RESULTADOS E DISCUSSÃO}

Inicialmente foram identificados os perfis dos pacientes participantes da pesquisa, para que fosse possível conhecer as características dos sujeitos que relataram sobre suas percepções sobre a dor. Primeiramente, foram coletados dados sociais para traçar um perfil dos pacientes internados (Tabela 1). Posteriormente, foram organizados em quadros e descritos em categorias temáticas, que emergiram da análise dos dados, sendo elas: Dor e Classificação da Dor. Assim, o perfil dos pacientes que participaram do estudo foram de ambos os sexos, maiores de 18 anos e que possuíam diagnóstico de Infarto Agudo do Miocárdio, estando clinicamente estáveis, conscientes e orientados e internados na Clínica Cardiológica, conforme o quadro a seguir.

A pesquisa em questão teve a participação de oito (8) pacientes com diagnóstico clínico de Infarto azgudo do Miocárdio com Supra de ST (IAM com Supra), internados na Clínica Cardiológica da Fundação Estadual Hospital de Clínicas Gaspar Viana. De acordo com os dados obtidos por meio de entrevista aos pacientes foi constatado que a maioria, ou seja, $62,5 \%$ (5) pacientes são do sexo feminino, caracterizando assim um maior acometimento de Infarto Agudo do Miocárdio (IAM) em mulheres.

Quanto aos fatores de risco relacionados aos antecedentes pessoais e familiares mórbidos (Tabela 2), os pacientes alegaram ter duas ou mais opções presentes no questionário, onde a maioria $75 \%$ (6) afirmou serem tabagistas, cinco (5) ou 40\% eram portadores de Hipertensão Arterial Sistêmica (HAS) e, seis (6) ou $75 \%$ afirmaram ter casos na famílias de IAM.

REAS/EJCH | Vol.Sup.21 | e547 | DOI: https://doi.org/10.25248/reas.e547.2019 Página 3 de 9 
Tabela 1 - Características sociais dos pacientes internados.

\begin{tabular}{lcc}
\hline \multicolumn{2}{c}{ DISTRIBUIÇÃO DE ÓBITOS SEGUNDO IDADE NEONATAL } \\
\hline VARIÁVEL & № & $\%$ \\
SEXO & & \\
Masculino & 03 & 37,50 \\
Feminino & 05 & 62,50 \\
& & \\
FAIXA ETÁRIA & & \\
$18-30$ anos & 00 & 00,00 \\
$31-40$ anos & 00 & 00,00 \\
$41-50$ anos & 00 & 00,00 \\
51-60 anos & 02 & 25,00 \\
>60 anos & 06 & 75,00 \\
& & \\
ESCOLARIDADE & & 12,50 \\
Analfabeto & 01 & 50,00 \\
Ens. fund. Inco. & 04 & 00,00 \\
Ens. Fund. Comp & 00 & 12,50 \\
Ens. Med. Inco. & 01 & 12,50 \\
Ens. Med. Comp. & 01 & 12,50 \\
Ens. Sup. Inco. & 01 & 00,00 \\
Ens. Sup. Comp. & 00 &
\end{tabular}

Fonte: Protocolo de pesquisa, 2015.

Segundo o autor Mussi et al (2014), os estudos acerca da prevalência de IAM no campo de análise com base na categoria gênero é ainda incipiente, e necessitam de aprofundamento por meio de pesquisas que poderão trazer benefícios para a prevenção da morbidade e mortalidade pela doença. Entretanto, alguns estudos evidenciaram que as mulheres diagnosticadas com IAM, comparadas aos homens, retardaram mais para procurar um serviço de saúde após o início dos sintomas. Desta forma, o gênero pode ser um fator que diferencia o comportamento de indivíduos quanto a doença e o uso dos serviços de saúde.

Em contrapartida resultados de uma pesquisa realizada pelo Instituto Dante Pazzanese de Cardiologia em São Paulo - SP, mostrou que $60 \%$ dos pacientes com IAM são homens, com média de idade em torno de 56 anos. Esta prevalência tem como justificativa o fato de que as mulheres procuram mais os serviços de saúde que os homens, e consequentemente, realizam mais exames preventivos (PEREIRA, DIAS e SANTOS, 2013).

Nesse sentido, percebe-se que os estudos sobre a influência dos gêneros acerca do diagnóstico de IAM ainda são insuficientes, visto que na literatura encontram-se divergências entre os autores quanto a prevalência da doença de acordo com o sexo. Frente a este fato, se faz importante os trabalhos de educação em saúde envolvendo as questões que viabilizem ambos os gêneros, levando em consideração a especificidade de cada um, no intuito de minimizar os comportamentos individuais que são prejudiciais à saúde.

Em relação a idade, $75 \%$ (6) pacientes se encontram na faixa etária acima de 60 anos, o que evidencia uma maior prevalência de indivíduos considerados idosos. Segundo a Sociedade Brasileira de cardiologia (2016), as doenças cardiovasculares apresentam expressiva morbimortalidade na população idosa. Nos Estados Unidos, os idosos constituem $13 \%$ da população, entretanto são responsáveis por $65 \%$ das 
hospitalizações por doença cardíaca. Cerca de $85 \%$ das mortes por IAM ocorrem na população idosa. Até os 65 anos a Doença Aguda Coronariana (DAC) é muito mais prevalente nos homens, mas a partir dos 80 anos sua prevalência é equivalente em ambos os sexos (NICOLAU et al, 2014).

Tabela 2 - Características clínicas dos pacientes internados.

\begin{tabular}{lll}
\hline \hline \multicolumn{2}{c}{ DISTRIBUIÇÃO DE ÓBITOS SEGUNDO IDADE NEONATAL } \\
\hline \multicolumn{1}{c}{ VARIÁVEL } & No & \\
PACIENTE CIRÚRGICO & 05 & 37,50 \\
Sim & 03 & 62,50 \\
Não & & \\
& & \\
PERÍODO DE INTERNAÇÃO & 04 & 00,00 \\
18-30 anos & 02 & 00,00 \\
31-40 anos & 01 & 00,00 \\
$41-50$ anos & 01 & 25,00 \\
51-60 anos & 00 & 75,00 \\
>60 anos & & \\
& & \\
ANTECEDENTES & & \\
FAMILIARES & 06 & 12,50 \\
Infarto Agudo do Miocárdio & 02 & 50,00 \\
Revascularização & 02 & \\
Nega & & \\
ANTECEDENTES PESSOAIS & & \\
HAS & 05 & 62,50 \\
Diabetes & 03 & 37,50 \\
Tabagismo & 06 & 75,00 \\
Etilismo & 06 & 75,00 \\
Drogas llícitas & 00,00 \\
\hline
\end{tabular}

Fonte: Protocolo de pesquisa, 2015.

A partir da identificação dos núcleos de sentido nos dados produzidos na pesquisa empírica, foi possível identificar duas categorias temáticas, por meio das quais serão descritos e discutidos os resultados encontrados. Iniciando com a categoria que descreve de que forma a dor foi iniciada.

\section{Categoria 1 - dor}

Esta categoria emergiu do questionamento sobre de que forma a dor foi iniciada, com objetivo de identificar se foi de forma repentina, ou após esforço físico, conforme demonstrado no quadro 3.

Durante as entrevistas realizadas com os pacientes foi levantado o questionamento acerca de que forma a dor iniciou, para identificar se foi de maneira abrupta ou insidiosa e quais são os fatores que iniciam ou intensificam esta dor. 
Quadro 1 - De que forma a dor iniciou?

\begin{tabular}{|l|}
\hline \multicolumn{1}{|c|}{ Unidade de Significado } \\
\hline - Após realizar atividades rotineiras (ex.: dormir, acordar, tomar café) \\
\hline - Após realizar esforço físico (ex.: caminhada) \\
\hline - Insidiosa, dor epigástrica inespecífica há muitos dias, e até anos. \\
\hline - Estresse \\
\hline - Dor na garganta e precordialgia após fumar \\
\hline
\end{tabular}

Fonte: Protocolo de pesquisa, 2015.

Para responder como a dor foi iniciada três (3) pacientes, relataram que os episódios iniciaram após realizarem atividade rotineiras como: dormir e tomar café. Como observamos no relato do Paciente 7:

"[...] Eu estava dormindo e acordei de repente com uma falta de ar muito forte, dor no estômago e vomitando muito."

Enquanto, dois (2) pacientes afirmaram que nunca tiveram indícios de dor, constando que a mesma se iniciou de forma abrupta. Em contrapartida dois (2) dos participantes da pesquisa relataram que sentiam dor precordial ou outro tipo de dor característica do infarto, como a dor na região cervical, há pelo menos 5 meses, sendo esta intensificada ao fumar, como refere o Paciente 6:

"[...]Há cinco meses eu sentia dor na garganta que piorava quando eu fumava e começa a dor meu peito, mas era uma dor suportável. Até que dia 23 de outubro de 2016, além da dor forte comecei a ter muita falta de ar, não aguentei e fui pra UPA da minha cidade, Castanhal-Pará. Lá não me deram remédio só colocaram oxigênio e me encaminhando pra cá (Hospital de Clínicas Gaspar Viana)."

Um (1) paciente afirmou que o estresse das rotinas do dia a dia ocasionou a dor precordial intensa, como relata o Paciente 5:

"[...]Meu dia a dia é muito estressante, me preocupo com os trabalhos de casa e os problemas na família, aí tive uma leve dor no peito dia 8 de setembro que não dei muita importância. Quando foi agora peguei um ônibus de Icoaraci pra Belém e começou a me dar uma queimação na boca do estômago, subindo pro peito, meu pescoço, braço esquerdo e ainda me deu muita falta de ar e tontura também. Aí me trouxeram direto pra cá (Hospital de Clínicas Gaspar Viana)."

Portanto, de acordo com os dados coletados, a unidade de significado que teve maior relevância foi a relacionada a dor que se inicia após a realização de atividades diárias. Este acontecimento pode muitas vezes mascarar os sintomas no paciente infartado, uma vez que ele pode relacionar a dor que está sentindo é a um mínimo esforço físico, retardando assim o início precoce dos cuidados médicos necessários.

A dor é definida como uma experiência sensorial e emocional desagradável e subjetiva associada a lesões reais ou potenciais e representa uma das principais causas de sofrimento humano. Dentre as dores de maior prevalência, destaca-se a dor torácica aguda, a qual é um dos motivos de maior procura pelos serviços de emergência. É um sintoma causado por várias doenças potencialmente fatais e tem um diagnóstico diferencial 
amplo. Entre eles estão incluídas as síndromes isquêmicas relacionadas no grupo das doenças cardiovasculares (SANTOS, VEIGA e ANDRADE, 2011). Segundo os mesmos autores a causa mais comum de dor torácica aguda consequente à isquemia do miocárdio é a doença coronariana aterosclerótica. Nesse cenário de dor torácica aguda, uma situação importante é a dor relacionada ao infarto agudo do miocárdio (IAM), desencadeada pela necrose do músculo cardíaco, provocada pela diminuição do fluxo de sangue no coração. Neste agravo, a dor torácica aguda constitui um importante sintoma e muitas vezes não tem diagnosticada a sua causa. Isso decorre da inexperiência e do conhecimento insuficiente no manejo de pacientes com dor, além da priorização em favor dos politraumatizados, dos com hemorragia digestiva, etc., no setor de emergência.

\section{Categoria 2 - classificação da dor}

Esta categoria emergiu dos questionamentos sobre de que forma o paciente infartado classifica a dor e como ele a descreve, com objetivo de identificar qual a percepção que eles possuem sobre o sintoma da dor e qual a principal característica definidora relacionada ao Diagnóstico de Enfermagem Dor Aguda.

Quadro 2 - Classificação da intensidade da dor.

\begin{tabular}{|c|}
\hline Unidade de Significado \\
\hline Fraca \\
\hline Moderada \\
\hline Forte \\
\hline Muito forte \\
\hline Insuportável \\
\hline
\end{tabular}

Fonte: Protocolo de pesquisa, 2015.

De acordo com a análise sobre como os pacientes classificam a dor foi possível perceber que houve uma prevalência entre a variável muito forte quatro e a variável insuportável quatro, deixando em evidência que a dor ocasionada pelo IAM é vivenciada pelo paciente de forma intensa, que interfere principalmente na realização de suas necessidades básicas, como respirar, o que evidencia como principal característica definidora do diagnóstico de Enfermagem: Mudanças no Parâmetro fisiológico. Como relata o paciente 7:

“[...]É uma dor insuportável. Não sei descrever certamente, mas eu não podia respirar, doía muito! Eu achava que ia morrer."

Três (3) pacientes descreveram a dor como se fosse algo que não tem explicação, que os levaram a vivenciar várias sensações, como algo que os aproximasse da morte. Observado no relato do paciente 5:

"[...]É uma dor insuportável, que queima dentro do coração, nem todas as dores são iguais e essa é muito diferente. É uma dor que parece que a morte tá chegando, agora eu sei que coração dói, de verdade."

Em outros dois (2) relatos os pacientes referiram-se a dor como se fosse algo muito forte, que poderia quebrar ou explodir o coração, como relata o paciente 8:

REAS/EJCH | Vol.Sup.21 | e547 | DOI: https://doi.org/10.25248/reas.e547.2019 Página 7 de 9 
"[...]É horrível! Uma dor que aperta o peito, que vem de dentro, como se o coração quisesse sair, como se fosse explodir o peito. A dor é muito horrível, achei que fosse morrer."

Segundo Carvalho apud Budó a dor é mais comumente vista como um sinal fisiológico que logo é sanado por terapêuticas medicamentosas, do que pela subjetividade permeada por suas sensações, em detrimento da cultura e crenças individuais. A ótica que cada indivíduo possui acerca da dor deve-se ao fato de que cada cultura atribui um significado a dor, segundo algumas crenças, é simbolizada como ato de resistência e coragem, como em cultos e rituais, outras vezes tida como uma manifestação de sofrimento, geralmente, associada a castigos divinos ou a enfermidades (CARVALHO e REZENDE, 2013).

A variedade dos tipos de dor deve-se as diversas causas de dor, a região em que ocorre o estímulo e a resposta com que cada indivíduo a infere. A resposta varia individualmente para um mesmo estímulo, pois cada estímulo doloroso recebe influência de mecanismos fisiológicos, psíquicos e socioculturais, assim, nem toda dor, necessariamente será acompanhada de sofrimento (CARVALHO, PAREJA e MAIA, 2015).

Segundo o autor Carvalho (2013), é importante que todo profissional da saúde, em especial a enfermagem, tenha conhecimento acerca do processo e dos mecanismos da dor, pois este é um dos maiores motivos de procura por assistência, além de um grande causador de transtornos físico-psicossosiais. Antes de implantar um tratamento é importante avaliar sistematicamente a pessoa e identificar os problemas, o que implicará em um tratamento mais bem sucedido, seguramente feito com base na anamnese e no exame físico do cliente.

Ainda segundo o autor Carvalho (2013), o enfermeiro contribui na avaliação da dor quando incorpora ao processo de enfermagem prescrições de cuidado para o seu alívio, medidas sejam elas farmacológicas ou não. Deve também avaliar a eficácia das ações/intervenções implantadas, monitorar a presença de efeitos adversos e atuar quando alguma intervenção prescrita se mostrar ineficaz. O campo de ações da enfermagem compreende atuar amplamente, embora em respeito a aceitação e a cultura individual.

\section{CONCLUSÃO}

A realização do estudo foi primordial para estabelecer o conhecimento acerca da percepção da dor do infarto que possuem os pacientes, para identificar quais as principais características que eles ressaltam, e para que isto aconteça o enfermeiro deve realizar seu papel de diagnosticador, através da utilização de seu conhecimento científico e raciocínio clínico, uma vez que o diagnóstico de enfermagem é de grande importância para estabelecer cuidados específicos a necessidade de cada paciente, visando além da melhora clínica, principalmente o seu bem estar no momento agudo da crise. Além de ratificar que de acordo com o que é encontrado na literatura, a definição e o enfrentamento da dor é algo pessoal e exclusivo de cada indivíduo. Mesmo que ambos passem por situações similares, cada um terá seu modo de expressar e descrever o significado da dor e o que ela representou no determinado momento que ocorreu. Entretanto, em relação a dor aguda ocasionada pelo Infarto Agudo do Miocárdio a percepção que os pacientes descreveram foi a mesma, em que a dor foi identificada como algo insuportável capaz de interferir na realização das atividades diárias básicas, classificando assim este tipo de dor como algo mais específico. Por este motivo se faz necessário o aperfeiçoamento dos profissionais da área da saúde, para que sejam capazes de diferenciar a dor do infarto das outras dores patológicas.

\section{REFERÊNCIAS}

1. BARDIN L. Análise de conteúdo/laurence bardin; tradução luís antero reto, agusto pinheiro. São Paulo/SP: Edições 70, 2011.

2. BRASIL, Datasus. Sistema de informações de mortalidade. óbitos por residência por ano do óbito segundo capítulo cid-10. dados preliminares de mortalidade. 2015.

REAS/EJCH | Vol.Sup.21 | e547 | DOI: https://doi.org/10.25248/reas.e547.2019 Página 8 de 9 
3. CARVALHO DC, PAREJA DCT, MAIA LFS. A importância das intervenções de enfermagem ao paciente com infarto agudo do miocárdio. São Paulo: Revista Recien. n. 3, v. 8, pag. 5-10. 2013.

4. CARVALHO FC, REZENDE ACC de. A enfermagem no cuidado ao paciente com dor: revisão de literatura. Rev CuidArte Enfermagem. n. 7, v. 2, pag. 119-123. 2013.

5. FALCÃO LF dos R, COSTA LHD, AMARAL LG do. Emergências fundamentos e práticas. 1ª Ed. 1139p. - São Paulo: Martinari, 2010.

6. FRANCELINO PC, OLIVEIRA LM de S, CARNEIRO NR, SIEBRA GM, BARBOSA IM, SALES DS. Assistência de Enfermagem ao Paciente com Infarto Agudo do Miocárdio: Revisão Integrativa. I Amostra do Internato de Enfermagem - Fortaleza, 2016.

7. MARKONI M de A, LAKATOS EM. Fundamentos de metodologia científica. 7. ed. rev. São Paulo: Atlas, 2010.

8. MUSSI FC, MENDES AS, DAMASCENO CA, GIBAUT, M de AM, GUIMARÃES AC, TELES CA de S. Fatores ambientais associados ao tempo de decisão para procura de atendimento no infarto do miocárdio. Acesso em 27 de dezembro de 2016. Disponível em: http://www.scielo.br/pdf/reben/v67n5/0034-7167-reben-67-05-0722.pdf

9. NICOLAU JC, Timerman A, Marin-Neto JA, Piegas LS, Barbosa CJDG, Franci A, Sociedade Brasileira de Cardiologia. Diretrizes da Sociedade Brasileira de Cardiologia sobre Angina Instável e Infarto Agudo do Miocárdio sem Supradesnível do Segmento ST. Arq Bras Cardiol. n. 102, v. 3, pag. 1-61. 2014.

10. OLIVEIRA MF, SILVA L de F. Enfermagem em laboratório de hemodinâmica: diagnóstico e intervenção fundamentados na Teoria da Adaptação de Roy. Rev. Eletr. Enf. [Internet]. n.12, vol. 4, pag. 678-85. 2010.

11. PEREIRA ACR, DIAS BVB, SANTOS FT dos S. Protocolo assistencial no pós infarto agudo do miocárdio baesado nos diagnósticos de enfermagem e intervenções da north american nursing diagnosis association NANDA. Rev CuidArte Enfermagem. n. 7, v. 2, pag. 113-118, 2013.

12. SANTOS N, VEIGA P, ANDRADE R. Importância da anamnese e do exame físico para o cuidado do enfermeiro. Rev Bras Enferm, Brasília, 2011.

13. SILVA JM, SILVEIRA ES. Apresentação de trabalhos acadêmicos: normas técnicas. 3. Ed. Petrópolis, RJ: Vozes, 2008.

14. VIEIRA AC. Protocolo de enfermagem para dor torácica em um serviço de emergência hospitalar: aplicação e avaliação. Florianópolis-SC, 2014. 